\title{
ArcheoSciences
}

Revue d'archéométrie

$31 \mid 2007$

Varia

\section{Tendances actuelles dans la caractérisation des obsidiennes pour les études de provenance}

Present trends in obsidian characterization for provenance studies

Gérard Poupeau, François-Xavier Le Bourdonnec, Stéphan Dubernet, Rosa B. Scorzelli, Mathieu Duttine et Tristan Carter

\section{OpenEdition}

1 Journals

Édition électronique

URL : https://journals.openedition.org/archeosciences/772

DOI : $10.4000 /$ archeosciences. 772

ISBN : 978-2-7535-1596-3

ISSN : $2104-3728$

Éditeur

Presses universitaires de Rennes

Édition imprimée

Date de publication : 31 décembre 2007

Pagination : 79-86

ISBN : 978-2-7535-0618-3

ISSN : $1960-1360$

Référence électronique

Gérard Poupeau, François-Xavier Le Bourdonnec, Stéphan Dubernet, Rosa B. Scorzelli, Mathieu

Duttine et Tristan Carter, «Tendances actuelles dans la caractérisation des obsidiennes pour les études de provenance », ArcheoSciences [En ligne], 31 | 2007, mis en ligne le 31 décembre 2009, consulté le 01 février 2022. URL : http://journals.openedition.org/archeosciences/772 ; DOI : https:// doi.org/10.4000/archeosciences. 772 


\title{
Tendances actuelles dans la caractérisation des obsidiennes pour les études de provenance
}

\author{
Present trends in obsidian characterization for provenance studies
}

\author{
Gérard Poupeau*/**, François-Xavier Le Bourdonnec*, Stéphan Dubernet*, \\ Rosa B. Scorzelli ${ }^{* * *}$, Mathieu DutTine*/*** et Tristan Carter ${ }^{* * * *}$
}

\begin{abstract}
Résumé : Les recherches de provenance des obsidiennes de sites paléolithiques et néolithiques connaissent actuellement une grande expansion, notamment par leur intégration dans les études de chaînes opératoires, qui nécessitent de déterminer l'origine de la matière première de séries importantes de pièces archéologiques. Il existe par ailleurs une forte demande de mesures peu à non destructives et éventuellement à effectuer in situ. D’où un certain nombre de développements méthodologiques récents. Le but de cette contribution est d'en présenter le statut actuel.

Abstract: Sourcing studies involving obsidians from Palaeolithic and Neolithic sites are presently undergoing a period of change, notably in terms of the average number of samples analyzed per project, due largely to their integration into the chaines opératoires approach of lithic technologies. This can entail working with a large number of artifacts from an archaeological context. There is also an ever-increasing demand to employ quasi non-destructive characterization methods and completely non-destructive methods that eventually could be performed in situ. As such, recent methodological advances have taken several directions. The purpose of this short note is to give an overview of these approaches.
\end{abstract}

Mots clés : Obsidienne, Composition élémentaire, Datation, Propriétés physiques, Provenance.

Key words: Obsidian, Obsidian elementary composition, Obsidian dating, Obsidian physical properties, Provenance.

\section{INTRODUCTION}

Les matériaux lithiques allochtones des sites préhistoriques constituent des indicateurs du mouvement des hommes et des matières premières qu'ils ont utilisées. L'obsidienne, que l'on rencontre parfois à des centaines, voire des milliers de kilomètres (Bird, 1991; Cauvin et Chataigner, 1998; Galipaud, 1998) de ses " sources " géo- logiques, compte parmi ces roches. Les recherches de « provenance " de ces obsidiennes ont débuté dans les années 1960 avec les articles fondateurs de Renfrew et collaborateurs sur les cultures néolithiques du bassin méditerranéen et des Proche et Moyen Orient (Cann et Renfrew, 1964; Renfrew et al., 1965, 1966, 1968). Pendant longtemps, ces travaux ont été menés en grande partie par des méthodes (partiellement) destructrices sur des déchets de taille, indé-

* CRP2A-IRAMAT, UMR 5060 CNRS - Université de Bordeaux, Maison de l'Archéologie, Esplanade des Antilles, 33607 Pessac, France. (gpoupeau@ u-bordeaux3.fr) (francois.lebourdonnec@etu.u-bordeaux3.fr) (Stephan.Dubernet@u-bordeaux3.fr)

** UMR 5198, Département de Préhistoire, Muséum national d'histoire naturelle, Musée de l'Homme, Place du Trocadéro 75007 Paris.

*** Centro Brasileiro de Pesquisas Fisicas, Rua Xavier Sigaud 150, 22290-180 Urca, Rio de Janeiro, Brésil. (scorza@cbpf.br, m.duttine@yahoo.fr)

**** McMaster University, Department of Anthropology, Chester New Hall, 524, 1280 Main Street West Hamilton, ON L8S 4L9, Canada. (stringy@ momaster.ca) 
pendamment des études de chaînes opératoires. Le plus souvent, le nombre de pièces analysées était relativement faible au regard du nombre de pièces collectées (voir par exemple Cauvin et al., 1998).

On tend actuellement à intégrer les études de provenance dans la problématique plus générale des chaînes opératoires et de l'économie des matières premières. Il faut pour cela procéder à des études exhaustives de séries lithiques et donc, pour les recherches de provenance, pouvoir traiter de grands nombres d'objets, dont certains de manière non destructive (Pereira et al., 2001; Joyce et al., 2004; Carter et al., 2005, 2006; Lugliè et al., 2006). D'où une recherche de nouvelles méthodes de caractérisation, rapides, peu coûteuse, très peu ou strictement non destructives. Le but de cette contribution est d'en présenter brièvement l'état actuel (voir aussi Liritzis, 2003; Poupeau et al., 2006).

\section{L'OBSIDIENNE}

L'obsidienne compte parmi les roches volcaniques les plus " acides ", c'est-à-dire les plus riches en silice $\left(\mathrm{SiO}_{2}\right.$ $>65 \%$ en masse). Elle est aussi, avec moins de $5 \%$ de cristaux en volume, l'une des plus " vitreuses ". Sous forme effusive, elle se présente en ségrégations litées ou en masse plus ou moins compactes dans des laves rhyolitiques. On la rencontre aussi parmi des produits d'origine pyroclastique, où elle apparaît essentiellement sous forme de nodules inclus au sein de nappes ignimbritiques (Hughes et Smith, 1993). Jusqu'à présent, les recherches de provenance des obsidiennes préhistoriques ont presque exclusivement reposé sur la spécificité de la composition chimique de chaque obsidienne-source (Cauvin et al., 1998; Shackley, 1998a). Bien que des variations intra-sources puissent parfois être importantes (Bellot-Gurlet et al., 2007), les différences entre sources sont en général suffisantes pour ne pas obérer les recherches de provenance. De plus, l'obsidienne étant une roche essentiellement vitreuse, ses caractéristiques physico-chimiques sont en principe semblables jusqu'à une échelle permettant des analyses "ponctuelles ». On connaît cependant de rares cas de convergences de composition élémentaire entre obsidiennes de sources relativement distantes au sein d'une même province volcanique (Bellot-Gurlet et al., 1999a), qui impliquent alors l'intervention d'autres moyens de discrimination, par l'âge de formation ou les propriétés physiques.

\section{Caractérisation par la géochimie}

Après les premiers travaux réalisés notamment par spectrométrie d'émission optique (Cann et Renfrew, 1964; Renfrew et al., 1966, 1968), deux méthodes donnant accès à des éléments traces discriminants ont rapidement dominé les recherches de provenance. Il s'est agit, dès la fin des années 1960, des analyses élémentaires partiellement destructives par activation neutronique instrumentale (INAA) (Gordus et al., 1967; 1968, Aspinall et al., 1972; Glascock et al., 1998), puis par fluorescence de rayons-X (XRF) (Francaviglia, 1984), voire non destructives par XRF (Shackley, 1998b) (Tableau 1). Une troisième méthode, l'analyse par faisceau d'ions (PIXE), largement utilisée depuis près d'une trentaine d'années pour suivre la progression de l'obsidienne de la Nouvelle Zélande vers le Pacifique central (Ambrose et al., 1981; Summerhayes et al., 1998), n'a vu son champ d'action s'accroître que vers la fin des années 1990 en Amérique andine (Poupeau et al., 1996; Bellot-Gurlet et al., 1999b) et plus récemment en Méditerranée (Poupeau et al., 2000; Le Bourdonnec et al., 2005a; Lugliè et al., 2007) et au Proche Orient (Abbès et al., 2003; Carter et al., 2007).

Dans certains cas toutefois, la seule détermination des teneurs en éléments majeurs peut se révéler déterminante. C'est ainsi que l'usage s'est répandu des analyses destructives par fluorescence-X en dispersion de longueur d'ondes avec une microsonde électronique (EMP-WDS) (Tykot, 1997, 2002; Le Bourdonnec et al., 2005b; Delerue, 2007). Il a aussi été montré que les analyses en dispersion d'énergie avec un microscope électronique à balayage (SEM-EDS), d'usage plus courant (Acquafredda et al., 1999), pouvaient représenter une alternative viable pour la Méditerranée occidentale (Acquafredda et al., 2006; Le Bourdonnec et al., 2006) et permettre un premier tri parmi les sources possibles dans les recherches de provenance au Proche Orient (Keller et Seifried, 1990; Delerue et al., 2007a).

Dans les années 1990, l'arsenal des méthodes de détermination des compositions élémentaires a vu apparaître deux innovations. D'une part, il s'agit des méthodes utilisant un plasma inductif, sous différentes formes, qui en permettant un dosage rapide de nombreux éléments, tendent à remplacer l'activation neutronique. Sous forme partiellement destructive, il s'agit d'analyses par spectroscopie d'émission atomique (ICP-AES) (Kilikoglou et al., 1997; Abbès et al., 2001; Bressy et al., 2005; Carter et al., 2006) ou spectrométrie de masse (ICP-MS) (Tykot, 1997; Abbès et al., 2001; Bressy et al., 2005; Carter et al., 2006; Delerue et al., 2007b). Dans ces configurations, l'analyse est souvent pratiquée sur une centaine de milligrammes, bien que techniquement un prélèvement de l'ordre du mg soit suffisant. 


\begin{tabular}{|c|c|c|c|}
\hline Méthode & Type* & «Consommation» typique & Éléments les plus fréquemment déterminés \\
\hline INAA & $\mathrm{D}$ & $100 \mathrm{mg}$ & $\begin{array}{l}\mathrm{Na}, \mathrm{K}, \mathrm{Ca}, \mathrm{Sc}, \mathrm{Cr}, \mathrm{Fe}, \mathrm{Co}, \mathrm{Ni}, \mathrm{Zn}, \mathrm{Ga}, \mathrm{As}, \mathrm{Se}, \mathrm{Rb}, \mathrm{Sr}, \mathrm{Zr}, \mathrm{Sb}, \mathrm{Cs}, \mathrm{Ba}, \\
\mathrm{La}, \mathrm{Ce}, \mathrm{Nd}, \mathrm{Sm}, \mathrm{Eu}, \mathrm{Gd}, \mathrm{Tb}, \mathrm{Ho}, \mathrm{Tm}, \mathrm{Yb}, \mathrm{Lu}, \mathrm{Hf}, \mathrm{Ta}, \mathrm{Th}, \mathrm{U}\end{array}$ \\
\hline ICP-AES & $\mathrm{D}$ & $100 \mathrm{mg}$ & $\mathrm{Na}, \mathrm{Mg}, \mathrm{Al}, \mathrm{P}, \mathrm{K}, \mathrm{Ca}, \mathrm{Ti}, \mathrm{Mn}, \mathrm{Fe}$ \\
\hline ICP-MS & $\mathrm{D}$ & $\begin{array}{l}\text { par voie humide : } 100 \mathrm{mg} \\
\text { par ablation laser : }<10^{-4} \mathrm{~mm}^{3}\end{array}$ & $\begin{array}{l}\text { Rb, Sr, Y, Zr, Nb, Cs, Ba, La, Ce, Pr, Nd, Sm, Eu, Gd, Tb, Dy, Ho, } \\
\text { Er, Yb, Lu, Hf, Ta, W, Pb, Th, U ( } \pm \text { Li, Mg, Sc, Ti, V, Mn, Co, Cu, } \\
\text { Zn, Ga, Sb, Tm, Tl) }\end{array}$ \\
\hline EMP-WDS & $\mathrm{D}$ & fragment millimétrique & $\mathrm{Na}, \mathrm{Mg}, \mathrm{Al}, \mathrm{Si}, \mathrm{P}, \mathrm{K}, \mathrm{Ca}, \mathrm{Ti}, \mathrm{Mn}, \mathrm{Fe}( \pm \mathrm{Cr}, \mathrm{Ba})$ \\
\hline SEM-EDS & $\mathrm{D}, \mathrm{ND}$ & fragment millimétrique (D) & $\mathrm{Na}, \mathrm{Al}, \mathrm{Si}, \mathrm{K}, \mathrm{Ca}, \mathrm{Fe}( \pm \mathrm{Ti}, \mathrm{Mn})$ \\
\hline EDXRF & ND & - & $\begin{array}{l}\text { Ti, Mn, Fe, Rb, Sr, Y, Zr, Nb, Ba ( } \pm \mathrm{Ni}, \mathrm{Cu}, \mathrm{Zn}, \mathrm{Ga}, \mathrm{La}, \mathrm{Ce}, \mathrm{Pr}, \mathrm{Pb} \text {, } \\
\text { Th) }\end{array}$ \\
\hline PIXE & ND & - & $\mathrm{Na}, \mathrm{Al}, \mathrm{Si}, \mathrm{K}, \mathrm{Ca}, \mathrm{Ti}, \mathrm{Mn}, \mathrm{Fe}, \mathrm{Zn}, \mathrm{Ga}, \mathrm{Rb}, \mathrm{Sr}, \mathrm{Zr}( \pm \mathrm{Mg}, \mathrm{Y}, \mathrm{Nb})$ \\
\hline \multicolumn{2}{|l|}{ INAA } & \multicolumn{2}{|l|}{ Instrumental Neutron Activation Analysis } \\
\hline \multicolumn{2}{|l|}{ ICP-AES } & \multicolumn{2}{|c|}{ Inductively Coupled Plasma-Atomic Emission Spectrometry } \\
\hline \multicolumn{2}{|l|}{ ICP-MS } & \multicolumn{2}{|c|}{ Inductively Coupled Plasma-Mass Spectrometry } \\
\hline \multicolumn{2}{|l|}{ EMP-WDS } & \multicolumn{2}{|c|}{ Electron Microprobe-Wavelength Dispersive X-Ray Spectrometry } \\
\hline \multicolumn{2}{|l|}{ SEM-EDS } & \multicolumn{2}{|c|}{ Scanning Electron Microscopy-Energy Dispersive X-Ray Spectrometry } \\
\hline \multicolumn{2}{|l|}{ EDXRF } & \multicolumn{2}{|c|}{ Energy Dispersive X-Ray Fluorescence Analysis } \\
\hline \multicolumn{2}{|l|}{ PIXE } & \multicolumn{2}{|c|}{ Particle Induced X-Ray Emission } \\
\hline
\end{tabular}

* D : Analyse destructive ; ND : Analyse non-destructive

Tableau 1 : Méthodes de détermination de la composition élémentaire des obsidiennes couramment utilisées dans les recherches de provenance.

Table 1: Methods of determination of obsidians elementary compositions in provenance studies.

Il est possible aussi de pratiquer des mesures quasiment non destructives si le prélèvement sur l'échantillon est réalisé par ablation laser (LA-ICP-MS) (Gratuze, 1999; Gratuze et al., 2001; Tykot, 2002; Carter et al., 2006). Dans ce cas, la " cicatrice " sur la pièce analysée se réduit à un cratère d'un diamètre inférieur à une centaine de microns.

\section{Caractérisation par l'âge de Formation}

Lâge de formation d'une obsidienne dans un édifice volcanique est atteignable par deux méthodes, les datations par traces de fission (fission track dating, FTD) ou par potassium-argon, dans sa déclinaison ${ }^{39} \mathrm{Ar} /{ }^{10} \mathrm{Ar}$. La première s'applique à toutes les obsidiennes formées il y a au moins environ 1000 ans, pourvu qu'elles soient transparentes en faible épaisseur $(<0,5-1 \mathrm{~mm})$, et dénuées d'artefacts observationnels (spurious tracks). Souvent discriminante, cette approche est d'application limitée parce qu'exigeante en matière (deux plaquettes de quelques $\mathrm{mm}^{2}$ chaque) et en temps d'acquisition de données (Poupeau et al., 1998). Elle a été très utilisée pour l'Amérique andine (Dorighel et al., 1998), la Méditerranée occidentale (Bigazzi et Radi,
1998) et les Proche et Moyen Orient (Bigazzi et al., 1998; Badalian et al., 2001). La datation par ${ }^{39} \mathrm{Ar} /{ }^{40} \mathrm{Ar}$, elle aussi destructive, ne nécessite qu'un prélèvement de l'ordre de $50 \mathrm{mg}$, mais c'est une méthode coûteuse et nécessitant une instrumentation très spécifique, qui n’a d'ailleurs été utilisée qu'exceptionnellement, là où d'autres approches s'étaient avérées insuffisantes (Vogel et al., 2006). La caractérisation par l'âge de formation, pour efficace qu'elle puisse parfois être, doit ainsi être réservée aux seuls cas d'indétermination par d'autres moyens (Bellot-Gurlet et al., 1999a; Dorighel, 2000 ; Dorighel et al., en préparation).

\section{Caractérisation PAR LES PROPRIÉTÉS PHYSIQUES}

Depuis les années 1990, plusieurs approches proposées une vingtaine d'années auparavant ont été revisitées. C'est ainsi qu'il a d'abord été montré que, pour les obsidiennes de Méditerranée centrale et occidentale, la spectroscopie Mössbauer $\mathrm{du}{ }^{57} \mathrm{Fe}$ pouvait prendre le relais des méthodes géochimiques (Scorzelli et al., 2001). Cependant, la faible teneur en Fe des obsidiennes, de l'ordre du pour cent pour 


\begin{tabular}{|c|c|c|c|}
\hline Méthode & Type* & «Consommation» & Caractérisation \\
\hline Mössbauer & $\mathrm{D}$ & $250 \mathrm{mg}$ & $\begin{array}{l}\text { Paramètres hyperfins, rapport } \mathrm{Fe}^{2+} / \mathrm{Fe}^{3+} \text {, présence de composés } \\
\text { magnétiques }\end{array}$ \\
\hline Magnétométrie SQUID & $\mathrm{D}$ & $30 \mathrm{mg}$ & $\begin{array}{l}\text { Force coercitive, susceptibilité magnétique, Aimantation } \\
\text { rémanente et à saturation }\end{array}$ \\
\hline ESR & $\mathrm{D}$ & $<10 \mathrm{mg}$ & $\begin{array}{l}\text { Nature et abondance relative des espèces para- et ferro- } \\
\text { magnétiques }\end{array}$ \\
\hline IR & $\mathrm{D}$ & $<5 \mathrm{mg}$ & $\begin{array}{c}\text { Structure du réseau silicaté } \\
\text { et }\end{array}$ \\
\hline Raman & ND & - & identification éventuelle inclusions minérales \\
\hline Mössbauer & & \multicolumn{2}{|c|}{ Spectroscopie Mössbauer du ${ }^{57} \mathrm{Fe}$} \\
\hline SQUID & & \multicolumn{2}{|c|}{ Superconducting Quantum Interference Device } \\
\hline ESR & & \multicolumn{2}{|c|}{ Electron Spin Resonance } \\
\hline IR & & \multicolumn{2}{|l|}{ Spectroscopie InfraRouge } \\
\hline Raman & & \multicolumn{2}{|l|}{ Microspectroscopie Raman } \\
\hline
\end{tabular}

* D : Analyse destructive; ND : Analyse non-destructive

Tableau 2 : Méthodes de caractérisation des obsidiennes par leurs propriétés physiques.

Table 2: Methods of obsidian characterization from their physical properties.

les variétés calco-alcalines et jusqu'à près de $10 \%$ pour les peralcalines exige des durées de mesures (jusqu'à 70 heures) et une quantité de matière (de l'ordre de $250 \mathrm{mg}$ ) qui rendent cette méthode peu attractive pour des mesures de routine. Par contre, la résonance de spin électronique (ESR) et la magnétométrie SQUID, qui permettent aussi la distinction des sources d'obsidienne de Méditerranée, se révèlent nettement moins exigeantes en matière (Tableau 2) et permettent des mesures plus rapides (respectivement quelques minutes et quelques heures) (Duttine et al., 2003; Stewart et al., 2003; Cernicchiaro et al., 2005; Duttine, 2005) et d'Equateur (Duttine et al., 2007). Il pourrait en être de même avec la spectroscopie InfraRouge en Méditerranée occidentale, comme le suggèrent quelques données préliminaires (Le Bourdonnec, 2003; Le Bourdonnec et al., 2003).

Alors que les méthodes précédentes nécessitaient un prélèvement, bien que parfois minime, sur les échantillons à traiter, la microspectroscopie Raman permet une caractérisation strictement non-destructive, à la fois de la minéralogie des obsidiennes et de la structure de la trame silicatée de sa phase vitreuse (Milleville et al., 2003). Un premier travail sur des obsidiennes méditerranéennes a montré de réelles potentialités pour cette méthode (Bellot-Gurlet et al., 2004), dont les applications pourraient cependant n'être limitées qu'à des cas particuliers en raison de durées de mesure encore très longues, de l'ordre de quelques heures.

\section{CONCLUSION : VERS DES STRATÉGIES ANALYTIQUES DIFFÉRENCIÉES?}

C'est donc de près d'une vingtaine de méthodes dont on dispose actuellement pour les études de provenance d'obsidiennes, de performance, de coûts analytiques et d'accessibilité divers. Selon les circonstances, région et fenêtre temporelle étudiées, possibilité ou non de procéder à des analyses partiellement destructives, telle méthode, ou telle combinaison de méthodes discriminantes pourrai (ent)t préférentiellement être adoptée(s), en fonction des problématiques archéologiques, des coûts induits, des temps d'appareils disponibles, etc., en particulier en ce qui concerne les signatures géochimiques.

Ainsi, pour le Mésolithique et le Néolithique de Méditerranée centrale et occidentale, bien qu'il ait été montré que des études de provenance peu destructives soient réalisables par ICP-MS (Tykot, 1997) voire LA-ICP-MS (Barca et al., 2007), il est beaucoup plus économique de les mener par une combinaison entre détermination visuelle et EMP-WDS (Tykot, 1997; Le Bourdonnec et al., 2005b) ou mieux, détermination visuelle et SEM-EDS (Le Bourdonnec et al., 2006). Si par contre l'intégrité des échantillons doit être intégralement respectée, c'est un couplage détermination visuelle/PIXE (Lugliè et al., 2006, 2007) ou détermination visuelle/XRF (Tykot et Ammerman, 1997) qui prendra l'avantage, voire éventuellement une association détermination visuelle/SEM-EDS, cette dernière combinaison demandant toutefois encore à être testée. De même, en 
Méditerranée orientale, alors que la distinction entre les deux sources d'obsidienne de Melos était basée sur de faibles mais significatives différences de teneur en scandium obtenues par INAA (Kilikoglou et al., 1997; Carter et Kilikoglou, 2007), les analyses par XRF non-destructives les séparent maintenant aisément par les teneurs en Ti et Sr (Liritzis, 2007). Il en est de même avec la résonance de spin électronique (Duttine et al., 2003; Duttine, 2005; Duttine, Villeneuve et Poupeau, en préparation), ces deux alternatives étant à la fois plus rapides et plus économiques que l'activation neutronique. Dans les Proche et Moyen Orient de l'Epipaléolithique et du Néolithique acéramique, si le SEM-EDS peut incontestablement apporter des données sur des régions-sources de provenance, voire sur quelques déterminations de sources, il doit impérativement être complété par des approches plus performantes, comme le PIXE ou l'ICP-MS (Delerue, 2007, Delerue et al., en préparation). C'est encore plus vrai à partir du VII ${ }^{e}$ millénaire avant notre ère, où les échanges s'intensifient et se diversifient. Les mesures par INAA, plus longues, plus lourdes, tendent à tomber en déshérence.

Si l'avenir des caractérisations par l'âge de formation des obsidiennes parait bien réservé aux cas insolubles par d'autres moyens (Bellot-Gurlet et al., 1999a; Vogel et al., 2006), celui des identifications de sources par les propriétés physiques est encore indéterminé. Bien que les mesures de résonance de spin électronique, de magnétisme, ou en spectroscopie InfraRouge soient rapides et ne nécessitent que très peu de matière, on manque encore de recul pour évaluer leur utilité réelle, malgré de premiers tests positifs, en particulier dans la mesure où l'état physique d'une obsidienne est susceptible de varier au sein d'une coulée, en fonction de l'histoire thermodynamique locale du magma qui lui a donné naissance. Spectroscopie Mössbauer et microspectroscopie Raman verraient de toutes façons leurs champs d'action très réduits, par l'exigence en matière (Mössbauer) et/ou en temps de mesure (Mössbauer, Raman).

La géochimie isotopique, non mentionnée jusqu'ici, est aussi un moyen puissant de discrimination entre obsidiennes de différentes sources. Cependant, malgré un essai positif réalisé par Gale (1981) avec le rapport ${ }^{87} \mathrm{Sr} /{ }^{86} \mathrm{Sr}$, ce type de caractérisation avait été par la suite délaissé pour les études de provenance, au profit de méthodes plus accessibles. Le développement des techniques ICP-MS et LA-ICP-MS pourrait cependant permettre bientôt un retour de cette approche.

Jusqu'ici, il n'a été question que d'objets analysés en laboratoire. Mais pour des raisons diverses (taille, fragilité, préciosité, etc.), il ne peut toujours en être ainsi, et c'est alors à l'expérimentateur à se déplacer jusqu'au musée, au site archéologique, etc., avec son appareillage. Dans un futur proche, ce sont donc les mesures effectuées in situ qui pourraient être privilégiées, comme en témoignent, pour les mesures par EDXRF, de premiers tests effectués avec des systèmes portables (cf. Carter et Shackley, 2007; Craig et al., 2007; Liritzis, 2007; Lopes et al., 2007).

\section{Bibliographie}

Abbès, F., Cauvin, M.-C., Gratuze, B., Bellot-Gurlet, L., Bressy, C. et Poupeau, G., 2001. Nouvelles recherches sur l'obsidienne de Cheikh Hassan (vallée de l'Euphrate, Syrie) au Néolithique: PPNA et PPNB ancien, Syria, 78, p. 5-17.

Abbès, F., Bellot-Gurlet, L., Cauvin, M.-C., Delerue, S., Dubernet, S., Poupeau, G. et Stordeur, D., 2003. Provenance of the Jerf el Ahmar (middle Euphrates valley, Syria) obsidians, Journal of Non-Crystalline Solids, 323, p. 162-166.

Acquafredda, P., Andriani, T., Lorenzoni, S. et Zanettin, E., 1999. Chemical characterization of obsidians from different Mediterranean sources by non-destructive SEM-EDS analytical method, Journal of Archaeological Science, 26, p. 315-325.

Acquafredda, P., Muntoni, I. M. et Pallara, M., 2006. La determinazione di provenienza dell' ossidiana mediante SEM+EDS: caratteristiche della metodica e casi studio dall' Italia sud-orientale. Materie prime e scambi nella Preistoria italiana, Atti della XXXIX Riunione Scientifica dell'Istituto Italiano di Preistoria e Protostoria, Florence, Istituto Italiano di Preistoria e Protoistoria, p. 509-519.

Ambrose, W. R., Duerden, P. et Bird, J. R., 1981. An archaeological application of PIXE-PIGME analysis to Admiralty Islands obsidians, Nuclear Instruments and Methods, 191, p. 397-402.

Aspinall, A., Feather, S. W. et Renfrew, C., 1972. Neutron activation analysis of Aegean obsidians. Nature, 237, p. 333334.

Badalian, R., Bigazzi, G., Cauvin, M. C., Chataigner, C., Jrbashyan, R., Karapetyan, S. G., Oddone, M. et Poidevin, J. L., 2001. An international research project on Armenian archaeological sites: fission-track dating of obsidians, Radiation Measurements, 34, p. 373-378.

Barca, D., De Francesco, A. M. et Crisci, G. M., 2007. Application of Laser Ablation ICP-MS for characterization of obsidian fragments from peri-Tyrrhenian area, Journal of Cultural Heritage, 8, p. 141-150.

Bellot-Gurlet, L., Bigazzi, G., Dorighel, O., Oddone, M., Poupeau, G. et Yegingil, Z., 1999a. The fission-track analysis: An alternative technique for provenance studies of prehistoric obsidian artefacts, Radiation Measurements, 31, p. 639-644.

ArcheoSciences, revue d'archéométrie, 31, 2007, p. 79-86 
Bellot-Gurlet, L., Poupeau, G., Dorighel, O., Calligaro, T., Dran, J.-C. et Salomon, J., 1999b. A PIXE/Fission-track dating approach to sourcing studies of obsidian artefacts in Colombia and Ecuador, Journal of Archaeological Science, 26, p. $855-860$.

Bellot-Gurlet, L., Le Bourdonnec, F.-X., Poupeau, G. et Dubernet, S., 2004. Raman micro-spectroscopy of western Mediterranean obsidian glass: one step towards provenance studies?, Journal of Raman Spectroscopy, 35, p. 671-677.

Bellot-Gurlet, L., Dorighel, O. et Poupeau, G., 2007. Obsidian provenance studies in Colombia and Ecuador: obsidian sources revisited, Journal of Archaeological Science, sous presse, corrected proof: doi:10.1016/j.jas.2007.03.008.

BigazZI, G. et RAdi, G, 1998. Prehistoric exploitation of obsidian for tool making in the Italian peninsula: a picture from a rich fission-track data set, Proceedings of the XIII International Congress of Prehistoric and Protohistoric Sciences, 1, p. 149156.

Bigazzi, G., Poupeau, G., Yegingil, Z. et Bellot-Gurlet, L., 1998. Provenance studies of obsidian artefacts in Anatolia using the fission-track dating method: an overview, in Cauvin, M.-C., Gourgaud, A., Gratuze, B., Arnaud, N., Poupeau, G., Poidevin, J.-L. et Chataigner, C. (éd.), L'Obsidienne au Proche et Moyen Orient, Du Volcan à l'Outil, Oxford, Archaeopress, p. 69-89.

BIRD, R. J., 1991. Obsidian characterisation and the peopling of the Pacific, in Menu, M., Walter, Ph. (éd.), La Pierre Préhistorique, Paris, Laboratoire de Recherche des Musées de France, p. 59-70.

Bressy, C., Poupeau, G. et Yener, K. A., 2005. Cultural interactions during the Ubaid and Halaf periods: Tell Kurdu (Amuq Valley, Turkey) obsidian sourcing, Journal of Archaeological Science, 32, p. 1560-1565.

CANN, J. R. et RenFrew, C., 1964. The characterization of obsidian and its application to the Mediterranean region, The Prehistoric Society, 30, p. 111-133.

Carter, T. et Kilikoglou, V., 2007. From reactor to royalty? Aegean and Anatolian obsidians from Quartier Mu, Malia (Crete), Journal of Mediterranean Archaeology, 20, p. 115145.

Carter, T., Poupeau, G., Bressy, C. et Pearce, N. J. G., 2005. From chemistry to consumption: towards a history of obsidian use at Çatalhöyük through a programme of inter-laboratory trace-elemental characterization, in Hodder, I. (ed.). Changing Materialities at Çatalhöyük: Reports from the 19951999 Seasons, Cambridge, McDonald Institute Monographs and BIAA, p. 285-305 et p. 535-557.

Carter, T., Poupeau, G., Bressy, C. et Pearce, N. J. G., 2006. A new programme of obsidian characterization at Catalhoyuk, Turkey, Journal of Archaeological Science, 33, p. 893-909.
Carter, T., Poupeau, G., Shackley, M. S., Dubernet, S., King, R. et Le Bourdonnec, F.-X., 2007. Eastern Anatolian obsidians at Çatalhöyük and the reconfiguration of regional interaction in the Early Pottery Neolithic, Antiquity, accepté.

Carter, T. et Shackley, M. S., 2007. Sourcing obsidian from Neolithic Çatalhöyük (Turkey) using energy dispersive X-ray Fluorescence, Archaeometry, 49, p. 437-454.

Cauvin, M.-C. et Chataigner, C., 1998. Distribution de l'obsidienne dans les sites archéologiques du Proche et Moyen Orient, in Cauvin M.-C., Gourgaud, A., Gratuze, B., Arnaud, N., Poupeau, G., Poidevin, J.-L., Chataigner, C. (éd.), L'Obsidienne au Proche et Moyen Orient, Du Volcan à l'Outil, Oxford, Archaeopress, p. 325-350.

Cauvin, M.-C., Gourgaud, A., Gratuze, B., Arnaud, N., Poupeau, G., Poidevin, J.-L. et Chataigner, C. (éd.), 1998. L'Obsidienne au Proche et Moyen Orient : du Volcan à l'Outil, Oxford, Archaeopress.

Cernicchiaro, G., Stewart, S. J., Scorzelli, R. B., Takeuchi, Y., Poupeau, G. et Bigazzi, G., 2005. Magnetic properties of obsidian and provenance studies in the Neolithic of Mediterranean basin, in Kars, H., Burke, E. (ed.), Geoarchaeological and Bioarchaeological Studies 3, Amsterdam, Vrije Universiteit, p. 61-63.

Craig, N., Speakman, R. J., Popelka-Filcoff, R. S., Glascock, M. D., Robertson, J. D., Shackley, M. S. et Aldenderfer, M. S., 2007. Comparison of XRF and PXRF for analysis of archaeological obsidian from southern Perú, Journal of Archaeological Science, sous presse, corrected proof: doi:10.1016/j.jas.2007.01.015.

Delerue, S., 2007. L'obsidienne dans le processus de néolithisation du Proche-Orient (12000-6500 BC), Thèse de doctorat, Université de Bordeaux, France.

Delerue, S., Poupeau, G., Dubernet, S., Balkan, N. et Molist, M., 2007a. Provenance of some obsidians from the Neolithic site of Akarçay (Sanliurfa, Turkey), in Molist, M. (éd.), Akarçay monograph, sous presse.

Delerue, S., Gopher, A., Milton, A., Poupeau, G. et Barrat, J.-A., 2007b. The Nahal Zehora II obsidians and their provenance, in Gopher, A. (ed.), Nahal Zehora II, a Pottery Neolithic site in Israël, sous presse.

Dorighel, O., 2000. La diffusion de l'obsidienne préhispanique dans l'aire andine équatoriale de 3500 BC à 1500 AD. Proposition d'une première modélisation des échanges par Traces de Fission et Géochimie, Thèse de doctorat, Université Panthéon-Sorbonne, Paris I, France.

Dorighel, O., Poupeau, G., Bellot-Gurlet, L. et Labrin, E., 1998. Fission track dating and provenience of archaeological obsidian artefacts in Colombia and Ecuador, in Van den Haute, P., de Corte, F. (éd.), Advances in Fission-Track Geochronology, Dordrecht, Kluwer Academic Publishers, p. 313-324. 
Duttine, M., 2005. Recherche de provenance de quartz et d'obsidiennes préhistoriques en Europe Occidentale. Apports de la Résonance Paramagnétique Electronique (RPE), Thèse de doctorat, Université de Bordeaux, France.

Duttine, M., Villeneuve, G., Poupeau, G., Rossi, A. M. et SCORZELLI, R.B., 2003. Electron spin resonance of $\mathrm{Fe}^{3+}$ ions in obsidians from Mediterranean islands. Application to provenance studies, Journal of Non-Crystalline Solids, 323, p. 193-199.

Duttine, M., Scorzelli, R. B., Cernicchiaro, G. R. C., Poupeau, G. et Guillaume-Gentil, N., 2007. Magnetic properties and electron spin resonance of Ecuadorian obsidians. Application to provenance research of archaeological samples, Journal of Magnetism and Magnetic Materials, sous presse.

Francaviglia, V., 1984. Characterization of Mediterranean obsidian sources by classical petrochemical methods, Preistoria Alpina, 20, p. 311-332.

GaLE, N. H., 1981. Mediterranean obsidian source characterisation by strontium isotope analysis, Archaeometry, 23, p. 41-51.

Galipaud, J.-C., 1998. Recherches archéologiques aux îles Torres, Journal de la Société des Océanistes, 107, p. 159-168.

Glascock, M. D., Braswell, G. E. et Cobean, R. H., 1998. A systematic approach to obsidian source characterization, in Shackley, M. S. (éd.). Archaeological Obsidian Studies. Method and Theory. Advances in Archaeological and Museum Science 3, New York/London, Plenum Press, 15-63.

Gordus, A. A., Fink, W. C., Hill, M. E., Purdy, J. C. et Wilcox, T. R., 1967. Identification of the geologic origins of archaeological artefacts: an automated method of $\mathrm{Na}$ and $\mathrm{Mn}$ neutron activation analysis, Archaeometry, 10, p. 87-96.

Gordus, A. A., Wright, G. A. et Griffin, J. B., 1968. Obsidian sources characterized by neutron-activation analysis, Science, 161, p. 382-384.

Gratuze, B., 1999. Obsidian characterization by laser ablation ICP-MS and its application to prehistoric trade in the Mediterranean and the Near East: sources and distribution of obsidian within the Aegean and Anatolia, Journal of Archaeological Science, 26, p. 869-881.

Gratuze, B., Blet-Lemarquand, M. et Barrandon, J. N., 2001. Mass spectrometry with laser sampling: a new tool to characterize archaeological materials, Journal of Radioanalytical and Nuclear Chemistry, 247, p. 645-656.

Hughes, R. E. et Smith, R. L., 1993. Archaeology, geology, and geochemistry in obsidian provenance studies, Geological Society of America, Special Paper 283, p. 79-91.

Joyce, R. A., Shackley, M. S., McCandless, K. et Sheptak, R., 2004. Resultados preliminares de una investigación con EDXRF de obsidiana de Puerto Escondido, in Yaxkin: Memoria, VII Seminario de Antropología de Honduras "Dr. George Hasemann", Honduras, Instituto Hondureño de Antropología e Historia, 115-130.
Keller, J. et Seifried, C., 1990. The present status of obsidian source identification in Anatolia and the Near-East, PACT, 25, p. 57-87.

Kilikoglou, V., Bassiakos, Y., Doonan, R. C. et Stratis, J., 1997. NAA and ICP analysis of obsidian from Central Europe and the Aegean: Source characterisation and provenance determination, Journal of Radioanalytical and Nuclear Chemistry, 216, p. 87-93.

Le Bourdonnec, F.-X., 2003. Caractérisation par PIXE, spectrométries Raman et InfraRouge d'obsidiennes. Application aux études de provenance d'artefacts néolithiques de la zone tyrrhénienne, Mémoire de DEA, Université de Bordeaux, France.

Le Bourdonnec, F.-X., Fröhlich, F., Poupeau, G. et Dubernet, S., 2003. Spectroscopie InfraRouge : un moyen d'étude de la provenance des obsidiennes néolithiques en Méditerranée occidentale?, in Livret de résumés du Colloque Spectroscopies vibrationnelles appliquées à la matière minérale : des gemmes aux verres et céramiques d'hier et de demain, Paris, Muséum National d'Histoire Naturelle, 43.

Le Bourdonnec, F.-X., Delerue, S., Dubernet, S., Moretto, P., Calligaro, T., Dran, J. C. et Poupeau, G., 2005a. PiXe characterization of Western Mediterranean and Anatolian obsidians and Neolithic provenance studies, Nuclear Instruments and Methods in Physics Research, Section B, 240, p. 595-599.

Le Bourdonnec, F.-X., Lugliè, C., Dubernet, S., Bohn, M., et Poupeau, G., 2005b. Monte Arci (Sardinia) obsidians: new geochemical data from electron microprobe and ion beam analysis, Atti del $3^{\circ}$ Convegno Internazionale L'ossidiana del Monte Arci nel Mediterraneo. Le vie dell'ossidiana nel Mediterraneo ed in Europa, Mogoro (Sardaigne), PTM, p. 129-140.

Le Bourdonnec, F.-X., Poupeau, G. et Lugliè, C., 2006. SEMEDS analysis of western Mediterranean obsidians: a new tool for Neolithic provenance studies, Comptes Rendus Geoscience, 338, p. 1150-1157.

LiRITZIS I. (éd.), 2003. Abstract book of the Workshop on Recent Advances in Obsidian Dating and Characterization, University of the Aegean, Laboratory of Archaeometry, 2-6 juillet.

LiRITZIS, I., 2007. Assessment of Aegean obsidian sources by portable ED-XRF analyser: grouping, provenance and accuracy, in Facorellis, Y., Zacharias, N., Polikreti, K. (éd.), Proceedings of the 4th HSA Symposium, Athens 2003, British Archaeological Reports Special Issue, sous presse.

Lopes, F., Melquiades, F. L., Appoloni, C. R., Parreira, P. S., Duttine, M., Poupeau, G. et Scorzelli, R. B., 2007. Characterization of Ecuadorian obsidians by X-ray fluorescence, in Livret de résumés, Primero Latino-Americano Simposio sobre Metodos Fisicos e Quimicos em Archeologia, Sao Paulo (Brésil), résumé.

Lugliè, C., Congia, C., Le Bourdonnec, F.-X., Bohn, M., Dubernet, S., Moretto, P., Sanna, I. et Poupeau, G., 2006.

ArcheoSciences, revue d'archéométrie, 31, 2007, p. 79-86 
Obsidian economy in the Rio Saboccu open-air Early Neolithic site (Sardinia), in Sternke, F., Costa, L. J., Eigeland, L. (éd.), Non-flint Raw Material Use in Prehistory: Old Prejudices and New Directions, Proceedings of the XVth Congress of the International Union of the Prehistoric and Protohistoric Sciences, Oxford, Archaeopress, sous presse.

Lugliè, C., Le Bourdonnec, F.-X., Poupeau, G., Atzeni, E., Dubernet, S., Moretto, P. et Serani, L., 2007. Early Neolithic obsidians in Sardinia (Western Mediterranean): the Su Carroppu case, Journal of Archaeological Science, 34, p. 428-439.

Milleville, A., Bellot Gurlet, L., Champagnon, B., et Santallier, D., 2003. La micro-spectroscopie Raman pour l'étude des obsidiennes : Structure, micro-inclusions, et études de provenance?, Revue d'Archéométrie, 27, p. 123-130.

Pereira, C. E. De B., Miekeley, N., Poupeau, G. et Küchler, I. L., 2001. Determination of minor and trace elements in obsidian rock samples and archaeological artifacts by laser ablation inductively coupled plasma mass spectrometry using synthetic obsidian standards. Spectrochimica Acta, Part B, 56, p. 1927-1940.

Poupeau, G., Bellot-Gurlet, L., Dorighel, O., Calligaro, T., Dran, J. C. et Salomon, J., 1996. Obsidian circulation in prehispanic times in Colombia and Ecuador: A coupled PIXE/fission track dating approach, Comptes Rendus de l'Académie des Sciences, Paris, Sciences de la Terre et des Planètes, 323, p. 443-450.

Poupeau, G., Bigazzi, G., Bellot-Gurlet, L. et Dorighel, O., 1998. Fission-track dating of obsidians and archaeology, in Cauvin, M.-C., Gourgaud, A., Gratuze, B., Arnaud, N., Poupeau, G., Poidevin, J.-L. et Chataigner, C. (éd.), L'Obsidienne au Proche et Moyen Orient, Du Volcan à l'Outil, Oxford, Archaeopress, p. 53-68.

Poupeau, G., Bellot-Gurlet, L., Brisotto, V. et Dorighel, O., 2000. Nouvelles données sur la provenance de l'obsidienne des sites néolithiques du Sud-Est de la France, Comptes Rendus de l'Académie des Sciences, Paris, Sciences de la Terre et des Planètes, 330, p. 297-303.

Poupeau, G., Le Bourdonnec, F.-X., Dubernet, S. et Delerue, S., 2006. Instrumental methods of obsidian characterization and prehistoric obsidian provenance studies: the current status, in Sternke, F., Costa, L. J., Eigeland, L. (ed.), Non-flint Raw Material Use in Prehistory: Old Prejudices and New Directions, Proceedings of the XVth Congress of the International Union of the Prehistoric and Protohistoric Sciences, Oxford, Archaeopress, sous presse.

Renfrew, C., Cann, J. R. et Dixon, J. E., 1965. Obsidian in the Aegean, Annual Report of the British School at Athens, 60, p. 225-247.
Renfrew, C., Dixon, J. E., et Cann, J. R., 1966. Obsidian and early cultural contact in the Near East, Proceedings of the Prehistoric Society, XXXII, p. 30-72.

Renfrew, C., Dixon, J. E., Cann, J. R., 1968. Further analysis of Near Eastern obsidians, Proceedings of the Prehistoric Society, 34, p. 319-331.

Scorzelli, R. B., Petrick, S., Rossi, A. M., Poupeau, G. et BigazzI, G., 2001. Obsidian archaeological artefacts provenance studies in the Western Mediterranean basin: an approach by Mossbauer spectroscopy and electron paramagnetic resonance, Comptes Rendus de l'Académie des Sciences, Paris, Sciences de la Terre et des Planètes, 332, p. 769-776.

Shackley, M. S. (éd.), 1998a. Archaeological Obsidian Studies: Method and Theory, London, Plenum Press.

SHACKLEY, S. M., 1998b. Geochemical differentiation and prehistoric procurement of obsidian in the Mount Taylor volcanic field, Northwest New Mexico, Journal of Archaeological Science, 25, p. 1073-1082.

Stewart, S. J., Cernicchiaro, G., Scorzelli, R. B., Poupeau, G., Acquafredda, P. et De Francesco, A., 2003. Magnetic properties and Fe-57 Mossbauer spectroscopy of Mediterranean prehistoric obsidians for provenance studies, Journal of NonCrystalline Solids, 323, p. 188-192.

Summerhayes, G. R., Bird, J. R., Fullagar, R., Gosden, C., Specht, J., et Torrence, R., 1998. Application of PIXEPIGME to achaeological analysis of changing patterns of obsidian use in the West New Britain, Papua New Guinea, in Shackley, M. S. (éd.), Archaeological Obsidian Studies. Method and Theory. Plenum Press, London, p. 129-158.

Түкот, R. H., 1997. Characterization of the Monte-Arci (Sardinia) obsidian sources, Journal of Archaeological Science, 24, p. $467-$ 479.

Түкот, R. H., 2002. Geochemical analysis of obsidian and the reconstruction of trade mechanisms in the Early Neolithic period of the western Mediterranean, in Jakes, K. (éd.). Archaeological Chemistry. Materials, Methods, and Meaning. ACS Symposium Series, American Chemical Society, Washington, D.C., p. 169-184.

Tүкот, R. H. et Ammerman, A. J., 1997. New directions in central Mediterranean obsidian studies. Antiquity, 71, p. 1000-1006.

Vogel, N., Nomade, S., Negash, A. et Renne, P. R., 2006. Forensic ${ }^{40} \mathrm{Ar} /{ }^{39} \mathrm{Ar}$ dating: a provenance study of Middle Stone Age obsidian artifacts from Ethiopia, Journal of Archaeological Science, 33, p. 1749-1765. 